\title{
Derivation of the Probability Distribution of Extreme Values of Offshore Structural Response by Efficient Time Simulation Method
}

\author{
M.K. Abu Husain*,1, N.I. Mohd Zaki ${ }^{1}$ and G. Najafian ${ }^{2}$ \\ ${ }^{1}$ UTM Razak School of Engineering and Advanced Technology, Universiti Teknologi Malaysia, Jalan Semarak, 54100 \\ Kuala Lumpur, Malaysia \\ ${ }^{2}$ School of Engineering, University of Liverpool, Brownlow St., Liverpool, L69 3GQ, UK
}

\begin{abstract}
Offshore structures are exposed to random wave loading in the ocean environment and hence the probability distribution of the extreme values of their response to wave loading is required for their safe and economical design. To this end, the conventional simulation technique (CTS) is frequently used for predicting the probability distribution of the extreme values of response. However, this technique suffers from excessive sampling variability and hence a large number of simulated response extreme values (hundreds of simulated response records) are required to reduce the sampling variability to acceptable levels. A more efficient method (ETS) was recently introduced which takes advantage of the correlation between the extreme values of surface elevation and their corresponding response extreme values. The method has proved to be very efficient for high-intensity sea states; however, the correlation and hence the efficiency and accuracy of the technique reduces for sea states of lower intensity. In this paper, a more efficient version of the ETS technique is introduced which takes advantage of the correlation between the extreme values of the nonlinear response and their corresponding linear response values.
\end{abstract}

Keywords: Offshore structure, Response extreme values, Morison's equation, Conventional Time Simulation.

\section{INTRODUCTION}

Offshore structures are subject to a wide variety of environmental loads (such as wind, wave, gravity and current) all of which exhibit a high degree of statistical uncertainty. However, in most cases, the dominant load is due to windgenerated random waves. Thus, the capability to predict the long-term probability distribution of the response extreme values is of great value to designers. The major obstacle in establishing the probabilistic properties of response is due to the nonlinearity of the wave load mechanism and/or the structural system which leads to non-Gaussian distribution for response [1-7]. The problem is further compounded by current and by intermittent loading on members in the splash zone, which have a significant effect on the statistical properties of extreme responses [8,9].

Many different techniques have been introduced for evaluation of statistical properties of response (refer to [3] for a brief partial review). In general, the probabilistic properties of response can be developed in the time, frequency or probability domains. In each case, sea-states are characterised by an appropriate water surface elevation spectrum, covering a wide range of frequencies. Conventional time simulation (CTS), finite-memory nonlinear system (FMNS)

\footnotetext{
*Address correspondence to this author at the UTM Razak School of Engineering and Advanced Technology, Unuversiti Teknologi Malaysia Kuala Lumpur, Jalan Semarak, 54100 Kuala Lumpur, Malaysia;

Tel: +603 2203 1385; Fax: +603 21805380 ;

E-mail: mohdkhairi@ic.utm.my
}

and NewWave theory are examples of time domain techniques. The principal component technique is a very efficient method for simulation of statistically independent quasistatic response values. In the frequency domain, Volterra series for derivation of higher-order spectra (bi-spectra and tri-spectra) of structural response have been implemented, and in the probability domain, procedures for derivation of the first four statistical moments of quasi-static response from the statistical properties of water particle kinematics have been introduced. The majority of the foregoing techniques are either very time consuming (such as the conventional time simulation technique) or limited in their application to special cases; for example, they cannot account for the effect of current or load intermittency in the splash zone, or are only applicable to quasi-static responses. In reality, the most versatile and reliable technique for predicting the statistical properties of the response of an offshore structure to random wave loading is the time domain simulation technique. However, this technique suffers from excessive sampling variability and hence a large number of simulated response extreme values (hundreds of simulated response records) are required to reduce the sampling variability to acceptable levels. If this technique can be speeded up, then it would be an ideal technique because it does not suffer from any of the inadequacies of the alternative methods and is applicable to all different types of structures.

An alternative time simulation method is based on the evaluation of the mean upcrossing rates of high levels of response. By considering an exponential model for the mean 
upcrossing rate of high response values, Naess et al [1] have succeeded in reducing the required number of simulated response records without sacrificing the accuracy of the predicted probability distribution of response extreme values. Another efficient version of the time simulation (ETS) technique has recently been introduced which takes advantage of the correlation between surface elevation and their corresponding response extreme values [10]. The method has proved to be very efficient for high-intensity sea states; however, its efficiency and accuracy reduces for sea states of lower intensity. This is due to the lower correlation between surface elevation and their corresponding response extreme values in the case of low-intensity sea sates, which, in turn, is believed to be due to shorter wavelengths for these sea states.

In this paper, a more efficient version of the ETS technique is introduced which takes advantage of the good correlation between the response and its corresponding linear response extreme values. This version of ETS technique had proved to be more accurate and efficient than the version based on surface elevation extreme values. Furthermore, both versions of the ETS technique have been used to calculate the long-term probability distribution of the response extreme values and have proved to be very effective. Again, the linear response version is somewhat more accurate. It should be noted that while this study accounts for the effect of load intermittency on members in the splash zone, it is limited to the case of quasi-static responses in the absence of current. The more general case of dynamic responses accounting for the effect of current is being investigated and the results would be published in due course.

The following section is devoted to a brief description of the test structure and selected responses. Morison's equation for evaluation of wave loading on cylindrical members of offshore structures is briefly discussed in Section 3. Then, a general outline of the simulation of a sample response record is presented in Section 4. Section 5 describes the CTS procedure for derivation of the probability distribution of response extreme values. This is followed by a brief description of the simulation of linear responses. The ETS procedure for derivation of the probability distribution of response extreme values is discussed in detail in Section 7. The accuracy of the ETS procedure for both short-term and long-term distributions of response extreme values is presented in Sections 8 and 9 , respectively. Finally, the conclusions of this study are summarized in Section 10.

\section{TEST STRUCTURE AND RESPONSES}

The test structure used in this study is a fixed platform in a water depth of $110 \mathrm{~m}$. The general outline of the platform is shown in Fig. (1). The platform is composed of four $1.5 \mathrm{~m}$ diameter vertical legs (similar to a jack-up platform) with a wall thickness of $40 \mathrm{~mm}$. As shown in the figure, the distributed hydrodynamic loading on each leg is represented by 30 point loads so that the total number of nodal loads on the four legs is 120 . The dimensions of the platform deck are $35 \mathrm{~m} * 38 \mathrm{~m}$. The member surfaces were assumed to be rough and hence the drag and inertia coefficients were taken to be
1.05 and 1.20 , respectively. For a more complete description of the test structure refer to [2].

The foregoing test structure was subjected to various unidirectional sea-states described by Pierson-Moskowitz ( $\mathrm{P}-$ $\mathrm{M})$ frequency spectrum. The waves were assumed to propagate in the global Y direction (Fig. 1). In this study, the following definition of the $\mathrm{P}-\mathrm{M}$ frequency spectrum [11] has been used:

$G_{\eta \eta}(f)=\frac{H_{S}{ }^{2}}{4 \pi T_{z}^{4} f^{5}} \exp \left(-\frac{1}{\pi T_{z}^{4} f^{4}}\right)$

where $f$ is the wave frequency in $\mathrm{Hz}, G_{\eta \eta}(f)$ is the surface elevation frequency spectrum, $H_{s}$ is the significant waveheight in meters and $T_{z}$ is the mean zero-upcrossing period in seconds.

Surface elevation and corresponding water particle kinematics at different nodes were simulated according to linear random wave theory (LRWT). All the water particle kine-

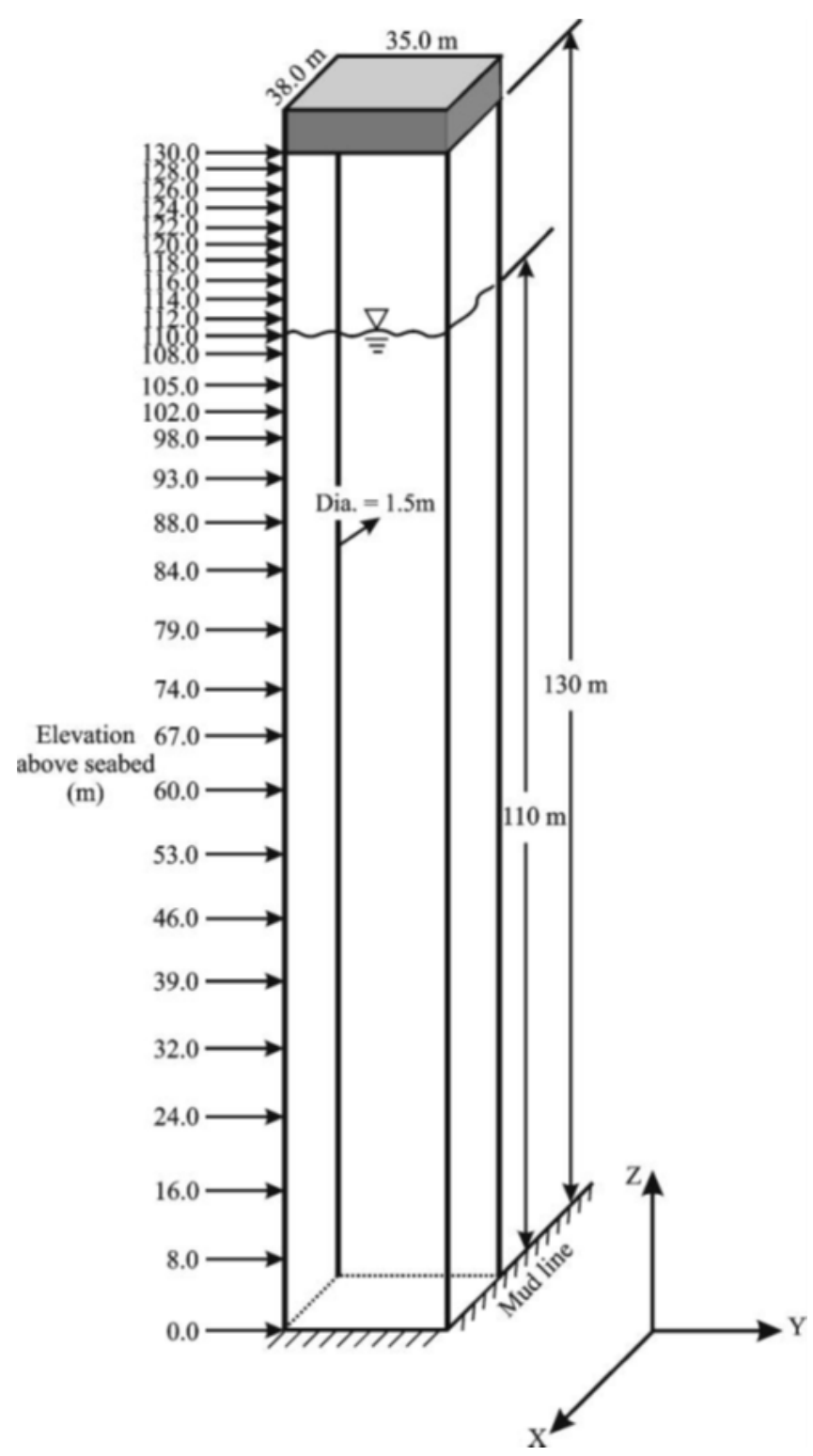

Fig. (1). Schematic diagram of the test structure. 
matics have been multiplied by a wave kinematics factor of 0.95 to account for wave directionality in the sea. The mean zero-upcrossing period (in seconds) for each sea state was taken to be $T_{z}=3.55 \sqrt{H_{s}}$ with $H_{s}$ denoting the significant wave height in meters. Furthermore, each response has been calculated for three different environmental conditions represented by $H_{s}=15 \mathrm{~m}, 10 \mathrm{~m}$, and $5 \mathrm{~m}$, respectively. Surface elevation frequency spectra for $H_{s}=15 \mathrm{~m}, 10 \mathrm{~m}$ and $5 \mathrm{~m}$ are shown in Fig. (2).

\section{WAVE LOADING ON CYLINDRICAL MEMBERS OF OFFSHORE STRUCTURES}

According to Morison's equation, the wave-induced horizontal force per unit length on a vertical submerged cylinder (cylinder diameter / wavelength < 1/5) is the sum of a nonlinear drag component and a linear inertial component. That is,

$F(t)=F_{\text {drag }}(t)+F_{\text {inertia }}(t)$

where drag and inertial components of fluid loading are, respectively, defined as

$F_{\text {drag }}(t)=k_{d} u(t)|u(t)|$

$F_{\text {inertia }}(t)=k_{i} \dot{u}(t)$

$k_{d}=\frac{1}{2} C_{d} \rho D$ and $k_{i}=\frac{1}{4} C_{m} \rho \pi D^{2}$

$C_{d}$ and $C_{m}$ are empirical drag and inertia coefficients; $\rho$ is the fluid density; $D$ is the leg cylinder diameter; and $u(t)$ and $\dot{u}(t)$ are the horizontal component of water particle velocity and acceleration, respectively. Further details about Morison's equation can be found in Sarpkaya and Isaacson [12] and Moe [13]. The assumption made in this paper is that Morison's equation with constant $C_{d}$ and $C_{m}$ values can adequately describe the in-line wave forces for a given sea state.

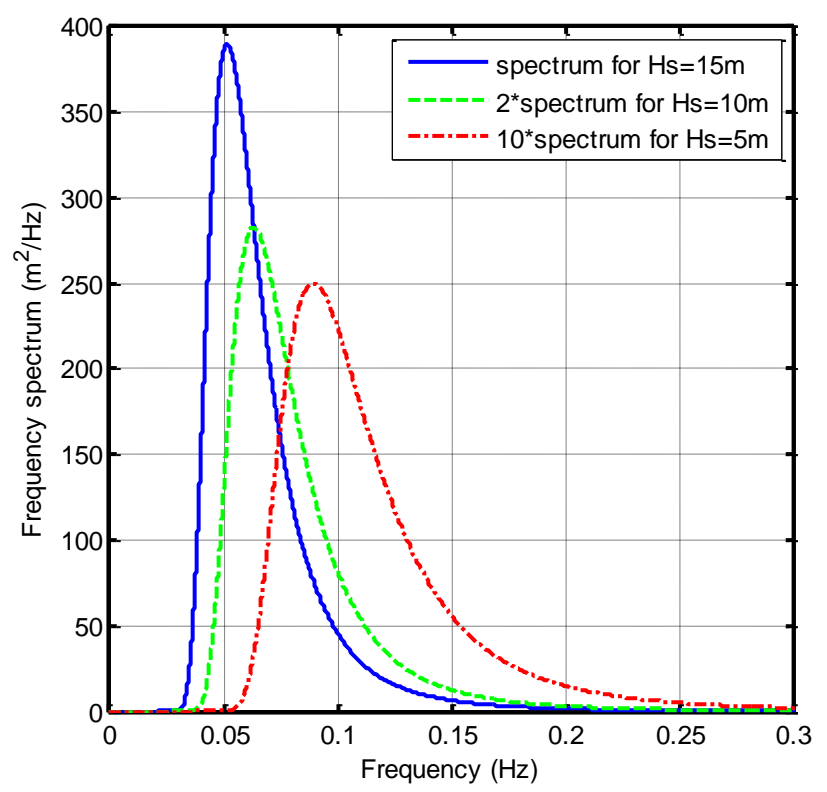

Fig. (2). Water surface elevation frequency spectra for three different sea states.

\section{EVALUATION OF RESPONSE RECORD BY CON- VENTIONAL TIME SIMULATION (CTS) PROCE- DURE}

The conventional time simulation (CTS) method is used in this study for the following two reasons: a) to calculate a transfer function for the linear response, and b) the probability distribution of extreme values of response from ETS method is compared with its corresponding probability distribution from the CTS procedure to investigate the accuracy and efficiency of the developed ETS models. Therefore, this section is devoted to a brief review of the procedure for simulation of a sample response record from the CTS method.

1. Use an appropriate surface elevation frequency spectrum such as Pierson-Moskowitz spectrum defined by its significant wave height $\left(H_{s}\right)$, and mean zeroupcrossing period $\left(T_{z}\right)$ [11].

2. Simulate surface elevation at an arbitrary reference point (in this study, at the centre of the platform) from the frequency spectrum for a given period of time using linear random wave theory (LWRT). According to LRWT, unidirectional seas can be modelled as the sum of a large number of linear progressive wavelets, all travelling in the same direction with random phase angles [14]. The surface elevation at point $x$ and time $t$ can then be expressed as:

$\eta(x, t)=\sum_{i=1}^{N W} A_{i} \cos \left(2 \pi f_{i} t-k_{i} x-\varphi_{i}\right)$

where $N W$ is the total number of wavelets used in the simulation, $f$ are a set of equally-spaced discrete wave frequencies, $k$ are their associated wave numbers, $\varphi$ are random phase angles distributed uniformly in the range 0 $<\varphi<2 \pi$, and finally $A$ are wave amplitudes, which can be determined from one of the following methods:

a. Deterministic Spectrum Amplitude (DSA) technique

$A_{i(D S A)}=\sqrt{2 G_{\eta \eta}\left(f_{i}\right) \Delta f}$

b. Non-Deterministic Spectrum Amplitude (NSA) technique

$A_{i(N S A)}=\sqrt{2 G_{\eta \eta}\left(f_{i}\right) \Delta f} * \sqrt{\frac{g_{i}{ }^{2}+h_{i}{ }^{2}}{2}}$

where in Eqs. (7) and (8), $G_{\eta \eta}$ is the one-sided surface elevation frequency spectrum and $\Delta f$ is the frequency interval. Parameters $g_{i}$ and $h_{i}$ are two independent and standardized Gaussian random variables. The NSA technique is more robust and hence was used in this study $[15,16]$.

3. Calculate wave-induced water particle kinematics at different nodes from the surface elevation record by using appropriate transfer functions from linear random wave theory as defined below.

$\Gamma_{\mathrm{u}}\left(f_{i}\right)=\omega_{i} \frac{\cosh \left(k_{i} z\right)}{\sinh \left(k_{i} d\right)} e^{-j k_{i}\left(x-x_{0}\right)}$

$\Gamma_{\dot{\mathrm{u}}}\left(f_{i}\right)=j \omega_{i} \Gamma_{\mathrm{u}}\left(f_{i}\right), j=\sqrt{-1}$

in which $\Gamma_{\mathrm{u}}$ and $\Gamma_{\dot{\mathrm{u}}}$ refer, respectively, to horizontal water particle velocity and acceleration transfer functions at a particular node; $x$ is the horizontal coordinate of the node and $z$ is its elevation above seabed. $\omega$ is the angular frequency $\left(\omega_{i}=2 \pi f_{i}\right), k$ is their associated wave numbers and $d$ is the water depth, which is the vertical distance 
from the mean water level (MWL) to the sea bed. $x_{0}$ is the horizontal coordinate of the arbitrary reference point at which the surface elevation has been simulated.

A common industry practice for evaluation of wave kinematics in the free surface zone consists of using linear wave theory in conjunction with empirical techniques to provide a more realistic representation of near-surface water kinematics. The empirical techniques popular in the offshore industry include Wheeler stretching, linear extrapolation, delta stretching, and vertical stretching. Couch and Conte [17] offer a review of these techniques. Vertical stretching [18] was adopted for this study. However, to avoid unrealistically large high frequency components for water particle kinematics in the vicinity of mean water level, a cut-off frequency of $0.30 \mathrm{~Hz}$ was considered for the surface elevation frequency spectrum.

4. Calculate the drag and inertial components of Morison force at each node accounting for load intermittency in the splash zone.

5. Calculate the quasi-static response from the Morison nodal loads. Assuming that the structural system is linear and that dynamic effects are negligible, the quasi-static base shear and overturning moment at the seabed can be calculated using the following equations:

Base shear, $B S=\sum_{i=1}^{N S}\left[F_{i} * \Delta l_{i}\right]$

Overturning moment, OTM $=\sum_{i=1}^{N S}\left[F_{i} * \Delta l_{i} * Z_{i}\right]$

where $N S$ is the number of nodal loads, $F_{i}$ is Morison load per unit length at node $i, \Delta l_{i}$ is the length of the element associated with node $i$, and $z_{i}$ is the elevation of node $i$ from seabed.

\section{DERIVATION OF PROBABILITY DISTRIBUTION OF RESPONSE EXTREME VALUES BY THE CON- VENTIONAL TIME SIMULATION (CTS) PROCE- DURE}

In this study, the probability distribution of response extreme values from the ETS technique will be compared with its corresponding distribution from the CTS procedure to examine the accuracy and efficiency of the developed ETS models. Thus, this section is devoted to a brief review of the derivation of the probability distribution of response extreme values from the CTS procedure.

\subsection{Short-term Probability Distribution of Response Extreme Values}

For short-term distribution, use the procedure in Section 4 to simulate a response record from a simulated surface elevation record and determine its extreme value. Then repeat the process many times to generate a large sample of response extreme values. Rank all the simulated extreme values from smallest to largest. Then use the following plotting position equation for the Gumbel distribution [16], to estimate the value of the probability distribution for each of the ranked extreme values.

$\operatorname{Prob}\left(r_{\max }<q_{n}\right)=P_{r_{\text {max }}}\left(q_{n}\right) \approx \frac{n-0.44}{N+0.12}$,

$n=1,2,3, \ldots, N$ where $r_{\max }$ denotes the response extreme value, $q_{n}$ is the $n$th smallest simulated extreme value, and finally $N$ is the total number of simulated extreme values.

As an example, the probability distribution of the extreme values of nonlinear quasi-static overturning moment of the test structure, plotted to the Gumbel scale, is shown in Fig. (3) for a sea state defined by $H_{s}=5 \mathrm{~m}$ and $T_{z}=7.94 \mathrm{sec}$. The plot is based on 20000 records, each of duration $T=128$ seconds.

\subsection{Long-term Probability Distribution of Response Ex- treme Values}

For design purposes, for example to calculate the value of the 100-year response, it is the long-term probability distribution of the response extreme values which is required. Therefore, it is the accuracy of the long-term distribution, which is of paramount importance. In this study the scatter diagram from the Forties Field (Table 1) has been used. The scatter diagram has been extended using the procedure described by Inglis et al. [19].

Now, $P_{L T}\left(r_{\max }\right)$, the long-term distribution of extreme response value accounting for all the sea states in the scatter diagram is derived in the following way [20],

$$
P_{L T}\left(r_{\text {max }}\right)=\sum_{i} \sum_{j} P_{r_{\text {max }}}\left(q_{n} \mid H_{s i}, T_{Z j}\right) * \frac{W_{i j}}{W}
$$

where $W_{i j}$ is the number of occurrences of the sea states represented by $H_{s i}$ and $T_{Z j}$ in the scatter diagram and $W=$ $\sum_{i} \sum_{j} W_{i j}$. In other words, $W_{i j} / W$ is the probability of occurrence of the sea state characterised by $H_{s i}$ and $T_{Z j}$. Examples of long-term distributions of response extreme values will be shown later.

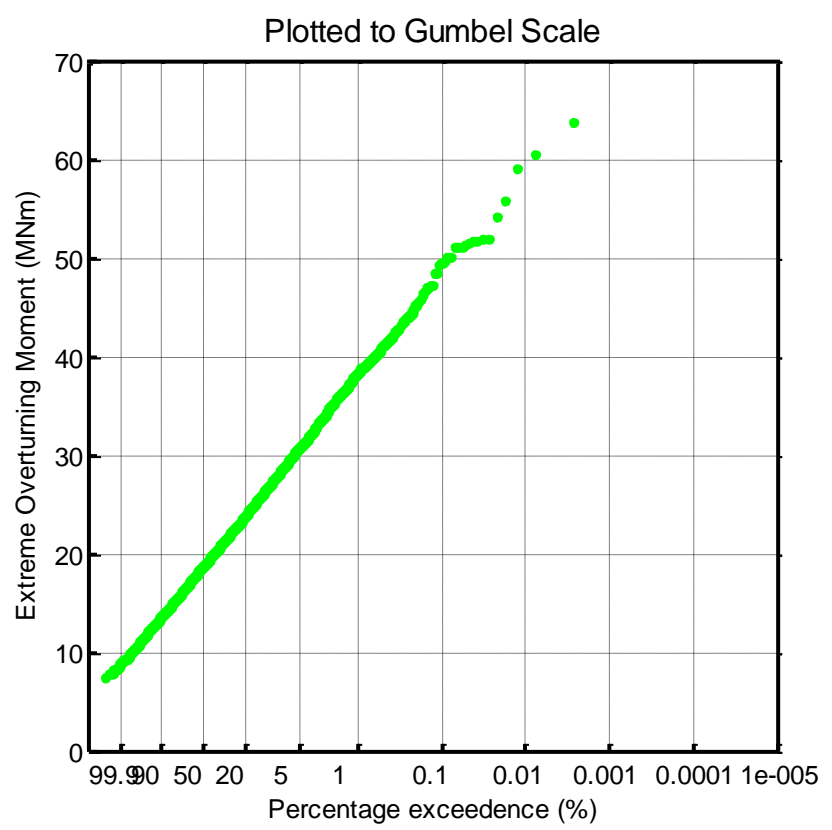

Fig. (3). Short-term probability distribution of extreme values of nonlinear quasi-static overturning moment; $H_{s}=5 \mathrm{~m}, T_{z}=7.94 \mathrm{sec}$, $T=128 \mathrm{sec}$, number of response records $=20000$. 
Table 1. Extended Scatter Diagram for Forties Field of the North Sea

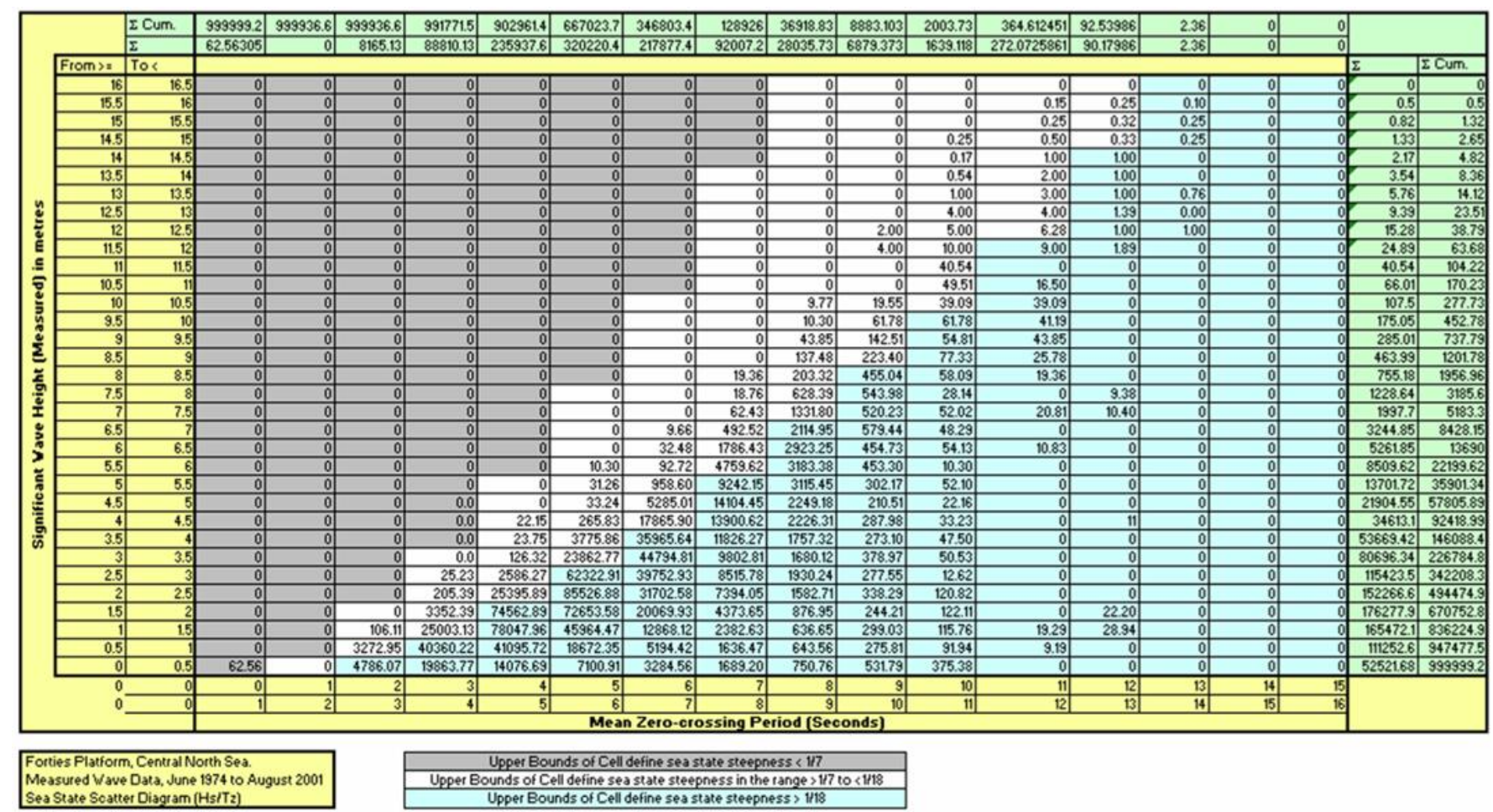

\section{SIMULATION OF LINEAR QUASI-STATIC RE- SPONSE}

As previously mentioned, the objective of this paper is to take advantage of the good correlation between nonlinear and linear response extreme values. In this section, derivation of linear quasi-static response is discussed.

Drag-induced and inertia-induced components of linear quasi-static response $\left(r_{L, \text { drag }}\right.$ and $r_{L, \text { inertia }}$, respectively) are defined as

$r_{\text {drag }} \approx r_{L, d r a g}=\alpha_{1} u_{1}+\alpha_{2} u_{2}+\alpha_{3} u_{3}+\cdots \alpha_{N S} u_{N S}$

$r_{\text {inertia }} \approx r_{L, \text { inertia }}=\beta_{1} \dot{u}_{1}+\beta_{2} \dot{u}_{2}+\beta_{3} \dot{u}_{3}+\cdots \beta_{N S} \dot{u}_{N S}$

where $u$ and $\dot{u}$ are, respectively, the horizontal component of water particle velocity and acceleration at each node. Eq. (16) implies that the relationship between the inertia-induced component of the quasi-static response and nodal water particle accelerations is not linear. The reason for nonlinearity is that in calculating the nonlinear quasi-static response, the effect of load intermittency on members in the splash zone has also been included. Therefore, to calculate the linear response, the linearization should be applied to both its draginduced and inertia-induced components.

The $\alpha$ and $\beta$ coefficients are determined through the least square error (LSE) method, by minimising $E\left[e^{2}\right]$ and $E\left[e^{\prime 2}\right]$ where $e$ and $e^{\prime}$ are defined as:

$$
\begin{aligned}
& e=r_{\text {drag }}-r_{L, \text { drag }} \\
& =r_{\text {drag }}-\alpha_{1} u_{1}+\alpha_{2} u_{2}+\alpha_{3} u_{3}+\cdots \alpha_{N S} u_{N S}
\end{aligned}
$$$$
\text { and }
$$

$e^{\prime}=r_{\text {inertia }}-r_{L, \text { inertia }}$

$=r_{\text {inertia }}-\beta_{1} \dot{u}_{1}+\beta_{2} \dot{u}_{2}+\beta_{3} \dot{u}_{3}+\cdots \beta_{N S} \dot{u}_{N S}$

Then, the transfer functions to convert the surface elevation to drag-induced and inertia-induced components of linear quasi-static response $\left(T F_{r_{L, d r a g}}\right.$ and $T F_{r_{L, \text { inertia }}}$, respectively) would be equal to.

$T F_{r_{L, d r a g}}=\sum_{i=1}^{N S} \alpha_{i} \Gamma_{\mathrm{u}, \mathrm{i}}$ and $T F_{r_{L, \text { inertia }}}=\sum_{i=1}^{N S} \beta_{i} \Gamma_{\mathrm{u}, \mathrm{i}}$

where $\Gamma_{\mathrm{u}, \mathrm{i}}$ and $\Gamma_{\dot{\mathrm{u}}, \mathrm{i}}$ are, respectively, the transfer functions of the horizontal components of water particle velocity and acceleration at the ith node. Then, in the case of a linear structure, the transfer function for the linear response would be the sum of the two foregoing transfer functions. That is,

$T F_{r_{L}}=\sum_{i=1}^{N S}\left(\alpha_{i} \Gamma_{\mathrm{u}, \mathrm{i}}+\beta_{i} \Gamma_{\dot{\mathrm{u}}, \mathrm{i}}\right)$

Once the transfer function of a linear response has been established, its simulation from a simulated reference surface elevation record is as straightforward as the derivation of a particle velocity or acceleration from the surface elevation.

\section{DERIVATION OF PROBABILITY DISTRIBUTION OF RESPONSE EXTREME VALUES FROM THE EF- FICIENT TIME SIMULATION (ETS) PROCEDURE}

The problem with the foregoing technique is that a large number of simulated extreme values are necessary to reduce the sampling variability to acceptable levels. The purpose of the proposed ETS procedure is to reduce the required number of simulated response records without sacrificing accuracy; hence, the excessive computer runtime can be reduced. In this section, a more efficient version of the ETS technique using extreme linear response magnitude discussed in details. It should be noted that a somewhat similar approach has already been discussed in [21], where advantage was 
taken of the good correlation between the extreme values of linear and nonlinear responses (the midspan moment of a fast-moving ship) to reduce the required time simulation for accurate derivation of the hourly maximum ship response statistics.

The correlation coefficients between simulated extreme values of linear and nonlinear quasi-static response (both base shear and overturning moment) for three different sea states $\left(H_{s}=15 \mathrm{~m}, 10 \mathrm{~m}\right.$ and $\left.5 \mathrm{~m}\right)$ are given in Table 2 . The table also gives corresponding correlation coefficients for surface elevation and (quasi-static) response extreme values. As observed, in all cases, and in particular for $H_{s}=5 \mathrm{~m}$, the correlation coefficients are higher for the linear response case. As an example, Fig. (4) shows the relationship between simulated extreme values of linear and nonlinear quasi-static overturning moment for $H_{s}=5 \mathrm{~m}, T_{z}=7.94$ and $T=128 \mathrm{sec}$, where $T$ is the duration of each simulated record.

Similarly, Fig. (5) shows the relationship between extreme values of surface elevation with extreme values of the quasi-static overturning moment. As observed, the correlation is stronger in the case of linear response extreme values. It is therefore expected that the ETS procedure based on linear response extreme values will perform better than the ETS method based on the surface elevation extreme values. The ETS method based on surface elevation extreme values has already been described in [10]. The version based on linear response extreme values is now explained.

\subsection{Theoretical Background}

The theoretical probability distribution of the linear quasi-static response extreme values during period $T$ (based on the assumption that the linear response is a Gaussian random process) is derived from the following relationship [16].

$$
\begin{aligned}
& \operatorname{Prob}\left(r_{L, \text { max }}<r_{L}\right)=P_{r_{L, \text { max }}}\left(r_{L}\right) \\
& =\exp \left[-M * \exp \left(-\frac{r_{L}^{2}}{2 \sigma_{r_{L}}^{2}}\right)\right]
\end{aligned}
$$

where $r_{L, \text { max }}$ denotes the linear response extreme value, $M=$ $T / T_{z}$ is the expected number of zero-upcrossings of the linear response during period $T$ and $\sigma_{r_{L}}$ is the standard deviation of linear response. As an example, the probability density function of the extreme values of the linear quasi-static overturning moment, for $H_{s}=5 \mathrm{~m}, T_{z}=7.94 \mathrm{sec}$ and $T=128 \mathrm{sec}$ is shown in Fig. (6). It is clear from the figure that the extreme values of the great majority of simulated records are between 5 and $25 \mathrm{MNm}$, and that linear quasi-static response records with very high extreme values are very rare. Therefore, con-

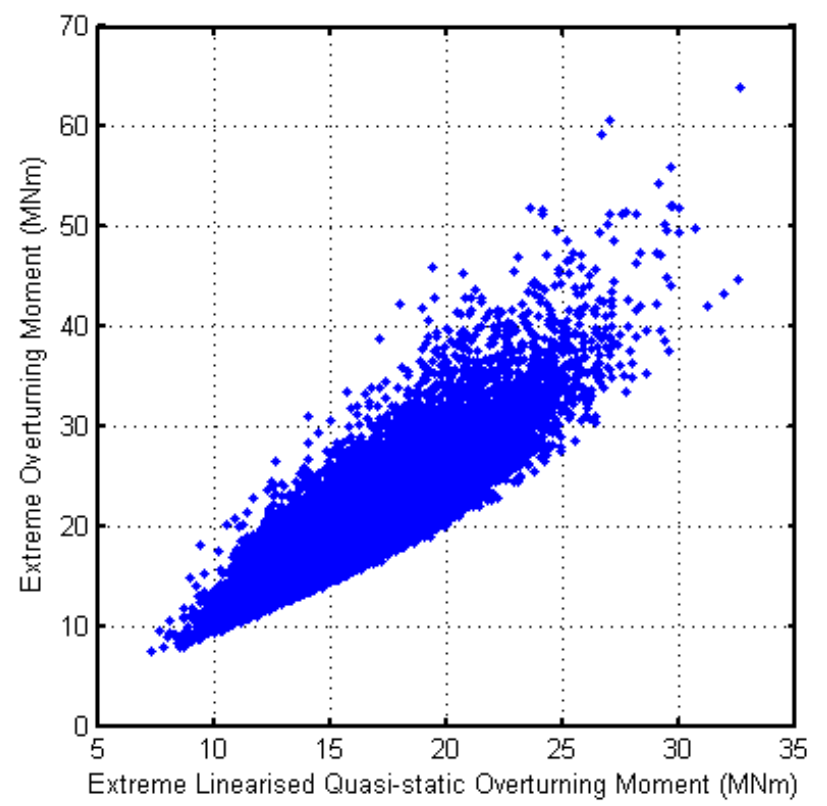

Fig. (4). Relationship between the extreme values of linear and nonlinear quasi-static overturning moment; $H_{s}=5 \mathrm{~m}, T_{z}=7.94 \mathrm{sec}$, $T=128 \mathrm{sec}$, number of response records $=20000$.

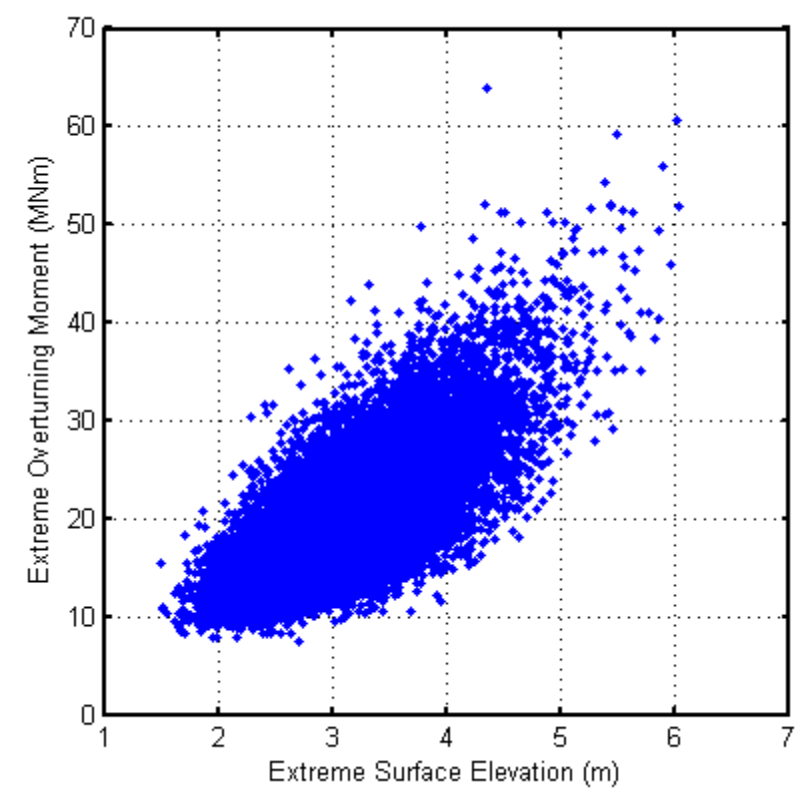

Fig. (5). Relationship between extreme surface elevation and extreme nonlinear quasi-static overturning moment; $H_{s}=5 \mathrm{~m}, T_{z}=$ $7.94 \mathrm{sec}, T=128 \mathrm{sec}$, number of response records $=20000$.

Table 2. Correlation Coefficients between Extreme Values of Simulated Nonlinear (Quasi-static) Response and Corresponding Surface Elevation Extreme Values in Comparison with Correlation Coefficients between Simulated Nonlinear and Linear (Quasi-static) Response Extreme Values (based on 20000 simulated records each of 128 sec duration)

\begin{tabular}{|c|c|c|c|c|c|c|}
\hline \multirow{2}{*}{ ETS Method } & \multicolumn{2}{|c|}{$\boldsymbol{H}_{s}=\mathbf{1 5 m}$} & \multicolumn{2}{|c|}{$\boldsymbol{H}_{s}=\mathbf{1 0 m}$} & \multicolumn{2}{|c|}{$\boldsymbol{H}_{s}=\mathbf{5 m}$} \\
\cline { 2 - 7 } & $\mathbf{B S}$ & OTM & BS & OTM & \multirow{2}{*}{ BS } & 0.723 \\
\hline \hline Surface Elevation extreme values & 0.916 & 0.912 & 0.883 & 0.709 \\
\hline Linear Response extreme values & 0.962 & 0.958 & 0.937 & 0.930 & 0.901 & 0.874 \\
\hline
\end{tabular}

Note: BS: Base shear and OTM: Overturning Moment 


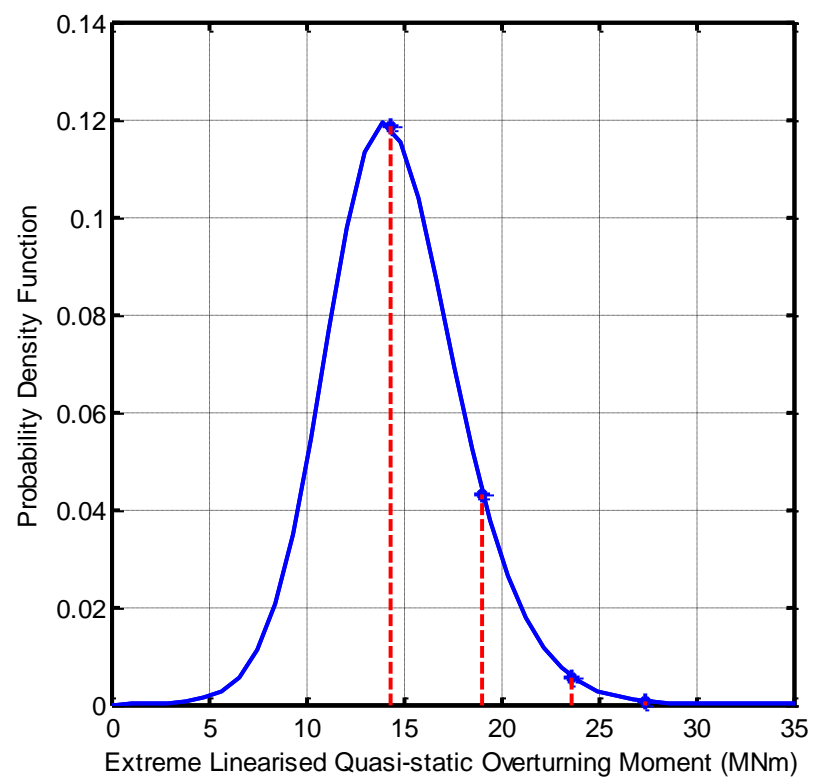

Fig. (6). Probability density function of extreme values of linear quasi-static overturning moment divided into 5 segments, $H_{s}=5 \mathrm{~m}$, $T_{z}=7.94 \mathrm{~s}, T=128 \mathrm{~s}$.

sidering the high correlation between the extreme values of linear and nonlinear quasi-static responses, it can be concluded that a large number of surface elevation records must be simulated and then converted to response records to get a fairly small number of response (both linear and nonlinear) records with high extreme values. This is the main problem with the CTS procedure.

To overcome the foregoing deficiency in the conventional time simulation (CTS) method, the ETS procedure has been introduced. In this method, the simulated response records are divided into a number of groups depending on the magnitude of the extreme value of their associated linear response record. In this study, five groups (refer to Fig. 6) and hence four different values of extreme linear quasi-static response have been considered corresponding to the following probability distribution values.

$P_{r_{L, \max }}\left(r_{L, i}\right)=\left[\begin{array}{llll}0.500 & 0.900 & 0.990 & 0.999\end{array}\right]$

The corresponding $r_{L, i}$ values for the above probability distributions can be determined from Eq. (21). For example, for $H_{s}=5 \mathrm{~m}, T_{z}=7.94 \mathrm{sec}$ and $T=128 \mathrm{sec}$, the linear quasistatic overturning moment $\left(r_{L, i}\right)$ values would be equal to

$r_{L, i}=[14.31 \mathrm{MNm} 19.03 \mathrm{MNm} 23.63 \mathrm{MNm} 27.40 \mathrm{MNm}](23)$

For the above example, the definition of each group and the probability of a simulated linear quasi-static response record and hence its corresponding nonlinear response record belonging to a particular group is given in Tables $\mathbf{3}$ and $\mathbf{4}$. Similar group definition for the efficient time simulation (ETS) procedure via surface elevation extreme values is given in Table 5. The expected number of simulated linear quasi-static response records and hence nonlinear quasi-static response records for each group can then be calculated by multiplying the total number of simulated records by the

Table 3. Definition of Groups Used in the Linear Response Version of the ETS Procedure for three Different sea States Applied to Quasi-static Base Shear

\begin{tabular}{|c|c|c|c|c|}
\hline $\begin{array}{c}\text { Group } \\
\text { Numberi }\end{array}$ & $\begin{array}{c}\text { Probability of } \boldsymbol{r}_{L, \max } \\
\text { belonging to each group } \\
\left(P_{i}\right)\end{array}$ & \multicolumn{3}{|c|}{ Definition of each group } \\
\hline 2 & 0.400 & $1.01 \mathrm{MN}<r_{L, \text { max }}<1.32 \mathrm{MN}$ & $0.51 \mathrm{MN}<r_{L, \max }<0.67 \mathrm{MN}$ & $0.19 \mathrm{MN}<r_{L, \text { max }}<0.24 \mathrm{MN}$ \\
\hline 3 & 0.090 & $1.32 \mathrm{MN}<r_{L, \max }<1.64 \mathrm{MN}$ & $0.67 \mathrm{MN}<r_{L, \text { max }}<0.82 \mathrm{MN}$ & $0.24 \mathrm{MN}<r_{L, \text { max }}<0.29 \mathrm{MN}$ \\
\hline 5 & 0.001 & $r_{L, \max }>1.89 \mathrm{MN}$ & $r_{L, \max }>0.94 \mathrm{MN}$ & $r_{L, \max }>0.33 \mathrm{MN}$ \\
\hline
\end{tabular}

Table 4. Definition of Groups Used in the Linear Response Version of the ETS Procedure for Three Different Sea States Applied to Quasi-static Overturning Moment

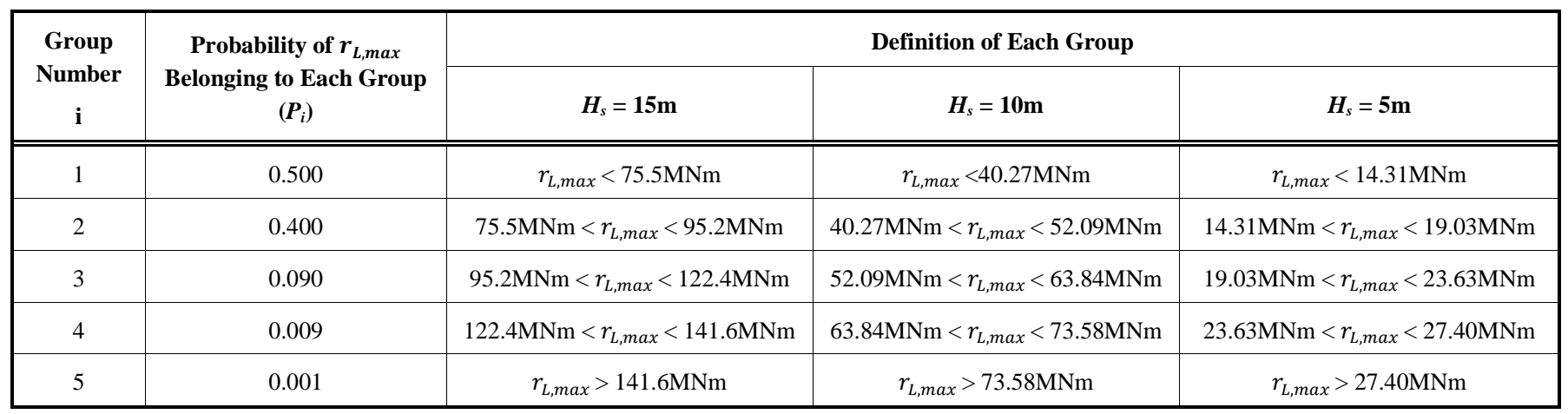


Table 5. Definition of Groups Used in Used in the Surface Elevation Version of the ETS Procedure for Three Different Sea States

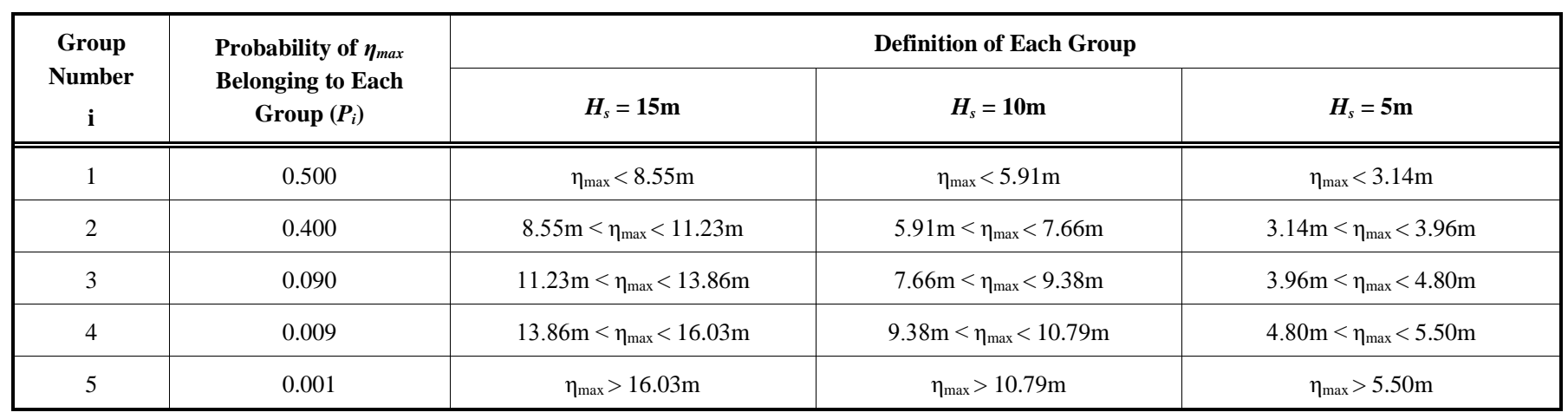

probability of occurrence of each group. As an example, for 20,000 simulated records, the expected number of linear quasi-static response records and hence nonlinear quasistatic response records belonging to Group 1 would be $20000 * 0.5=10000$ (refer to Table 6). It is clear from Table 6 that the great majority of simulated records belong to the first few groups and that only 20 records are expected to belong to the last group (the group with the highest linear quasi-static response extreme values and hence the highest nonlinear quasi-static response extreme values).

In the linear response version of the ETS technique, a surface elevation record is simulated and then converted to a linear quasi-static response record (using an appropriate transfer function as in Eq. 20). Then, this record is allocated to a particular group depending on the magnitude of its extreme value. The surface elevation record is then used to simulate a nonlinear quasi-static response record and its extreme value will be allocated to the same group. The process is repeated. When a group's target (for example, 50 for Group 1 of Case 1) has been achieved, then, further surface elevations records belonging to that group are discarded and are not converted into nonlinear quasi-static response records. The process is repeated until all groups have achieved their target numbers. This means that in practice, the great majority of simulated surface elevation records are not converted into nonlinear response records. In contrast, in the conventional time simulation (CTS) technique, simulated records are not divided into groups, and as a result, all the simulated surface elevation records must be converted to nonlinear response records to have an unbiased sample of response extreme values. This explains why the ETS procedure is much more efficient than the CTS procedure.

In the ETS procedure, the probability distribution of nonlinear quasi-static response extreme values for each group is calculated separately (see later), and then, the probability distribution of nonlinear response extreme values is derived by combining the probability distributions of the response extreme values for all groups. Further explanation follows.

Let $\mathrm{A}$ be the set of all response extreme values divided into $N G$ (mutually exclusive) groups based on the magnitude of the extreme values of their corresponding linear quasistatic response records (as previously explained). That is $\mathrm{A}=$ $\left\{\mathrm{A}_{1}, \mathrm{~A}_{2}, \mathrm{~A}_{3}, \ldots \mathrm{A}_{\mathrm{i}}, \ldots \mathrm{A}_{N G}\right\}$, where $\mathrm{A}_{\mathrm{i}}$ is the subset of nonlinear quasi-static response extreme values belonging to group i. Now using the total probability theorem, the probability distribution of the response extreme values can be calculated from the following relationship.

$$
\operatorname{Prob}\left(r_{\max }<q\right)=P_{r_{\max }}(q)=\sum_{i=1}^{N G} P_{r_{\max }}^{(i)}(q) * P_{i}
$$

where $P_{r_{\max }}^{(i)}(q)$ is the probability of the response extreme value being less than $q$ given that its corresponding linear response extreme value belongs to Group i. In other words, $P_{r_{\max }}^{(i)}(q)=P_{r_{\max }}\left(q \mid r_{L, \max } \in\right.$ Group i $)$, and $P_{\mathrm{i}}$ is the probability of occurrence of Group i (i.e. probability of linear response extreme value belonging to Group i). It should be noted that $P_{\mathrm{i}}$ values are already known; hence, according to Eq. (23), all that is required for accurate estimation of the

Table 6. Number of Simulated Records Used in Efficient Time Simulation (ETS) Procedure

\begin{tabular}{|c|c|c|c|c|}
\hline \multirow{2}{*}{$\begin{array}{l}\text { Group } \\
\text { Number } \\
\text { i }\end{array}$} & \multirow{2}{*}{$\begin{array}{c}\text { Probability of } \boldsymbol{\eta}_{\max } \text { and } \\
\boldsymbol{r}_{L, \max } \text { Belonging to Each } \\
\text { Group }\left(\boldsymbol{P}_{i}\right)\end{array}$} & \multirow{2}{*}{$\begin{array}{l}\text { Expected Number of Simulated Response Rec- } \\
\text { ords Practically Used for Each Group in the CTS } \\
\text { Procedure (based on } 20,000 \text { simulations) }\end{array}$} & \multicolumn{2}{|c|}{$\begin{array}{l}\text { Number of Simulated Response Records Used for } \\
\text { Each Group }\left(N_{\mathrm{i}}\right) \text { in the ETS Procedure }\end{array}$} \\
\hline & & & Case 1 & Case 2 \\
\hline 1 & 0.500 & 10000 & 50 & 10 \\
\hline 2 & 0.400 & 8000 & 50 & 10 \\
\hline 4 & 0.009 & 180 & 50 & 50 \\
\hline 5 & 0.001 & 20 & 20 & 20 \\
\hline Total & 1.000 & 20,000 & 220 & 100 \\
\hline
\end{tabular}


probability distribution of the response extreme values is the accurate estimation of $P_{r_{\max }}^{(i)}(q)$ for each group.

\subsection{Derivation of the Probability Distribution of the Re- sponse Extreme Values for Each Group}

The main point of the ETS technique is that there is no reason to calculate $P_{r_{\max }}^{(i)}(q)$ based on vastly different number of simulated extreme values for different groups (refer to Table 6, Column 3). This is, in effect, what is happening in the CTS procedure. Instead, from each group a limited number of surface elevation records $\left(N_{i}\right)$ are converted into nonlinear response records (Table 6, Columns 4 and 5). The probability distribution of response extreme values for each group (refer to Fig. (7), where for clarity, the distributions are plotted to the Gumbel scale) is then estimated from the following equation

$P_{r_{\text {max }}}^{(i)}\left(q_{n}^{(i)}\right)=\frac{n-0.44}{N_{i}+0.12}, n=1,2,3 \ldots, N_{i}$

where $q_{n}^{(i)}$ is the $n$th smallest response extreme value belonging to Group i. However, in order to calculate $P_{r_{\max }}(q)$ from Eq. (24), the values of $P_{r_{\max }}^{(i)}(q)$ must be known at all $q$ values, where $q$ is the set of all simulated response extreme values belonging to all the different groups. $P_{r_{\max }}^{(i)}(q)$ is calculated from the following equation.

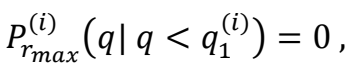

$P_{r_{\text {max }}^{(i)}}^{(q \mid q}\left(q q_{N_{i}}^{(i)}\right)=1$

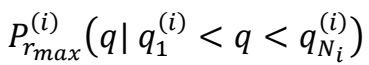

$=$ determine by interpolation from Eq. (25)

The validation of the efficient time simulation (ETS) technique follows.

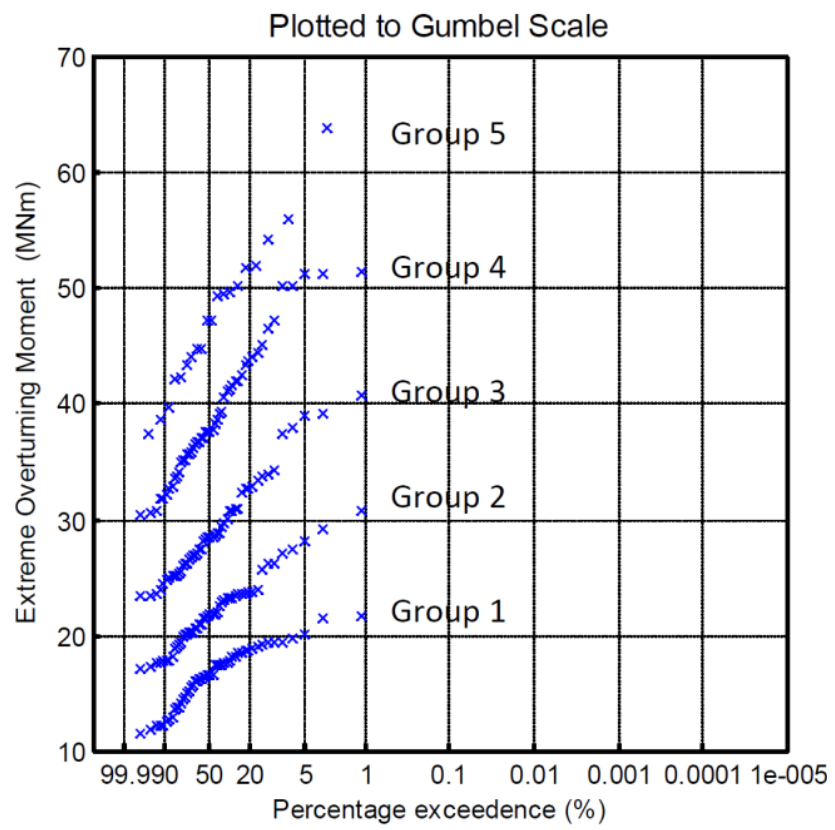

Fig. (7). Probability distribution of extreme values of response belonging to each group, $H_{s}=5 \mathrm{~m}, T_{z}=7.94 \mathrm{~s}, T=128 \mathrm{~s}$.

\section{COMPARISON OF THE TWO ALTERNATIVE VERSIONS OF THE ETS PROCEDURE: SHORT- TERM PROBABILITY DISTRIBUTION OF RE- SPONSE EXTREME VALUES}

In this section, the probability distribution of the extreme values of response from both versions of the ETS procedure are compared with those derived from extensive simulation using the CTS procedure to establish their accuracy and efficiency. In terms of the number of simulated records for the ETS procedure, two cases have been considered as defined in Table 6 (Columns 4 and 5, respectively). For Case 1, 50 simulated response extreme values have been allocated to each of the first 4 groups and 20 to the last group. For Case 2, 10 simulated response extreme values have been allocated to each of the first 3 groups, and groups 4 and 5 are similar to Case 1 (i.e., 50 and 20 records, respectively).

The probability distributions of the extreme values of nonlinear quasi-static response for Case 1 (based on a total number of 220 simulations, refer to Table 6 ) and for three different sea states $\left(H_{s}=15 \mathrm{~m}, 10 \mathrm{~m}, 5 \mathrm{~m}\right)$ are shown in Figs. (8-10), respectively. As observed, in all cases, the linear response version of the ETS procedure is in better agreement with corresponding distributions from the CTS procedure (CTS based on 20000 simulations). This can also be seen in Tables $\mathbf{7}$ and $\mathbf{8}$, where the extreme values at $0.1 \%$ probability of exceedence from the two alternative versions of the ETS procedure are compared with corresponding values from the CTS procedure. Again, as observed, the linear response version of the ETS procedure is more accurate.

Table 9 gives the ratios between the standard deviations of the inertia-induced and drag-induced components of response. Not surprisingly, the drag-induced component of response is more dominant for $H_{s}=15 \mathrm{~m}$, and the inertial component of response gradually becomes more important as $H_{s}$ decreases. Figs. (8-10) also give the distribution of linear

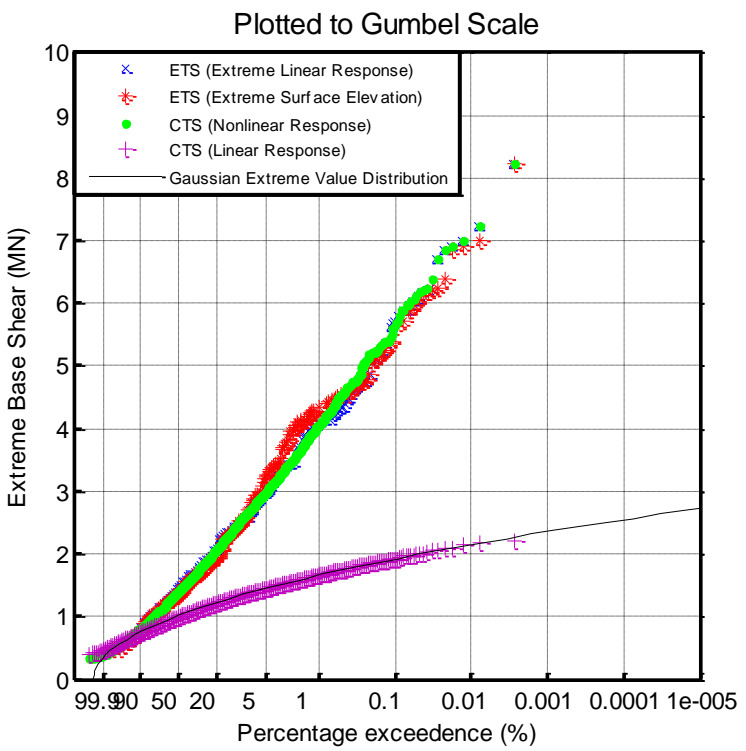

Fig. (8). Comparison of short-term probability distribution of extreme values of quasi-static base shear from ETS method (Case 1, 220 simulations) with corresponding distribution from CTS (20000 simulations), $H_{s}=15 \mathrm{~m}, T_{z}=13.75 \mathrm{~s}, T=128 \mathrm{~s}$. 


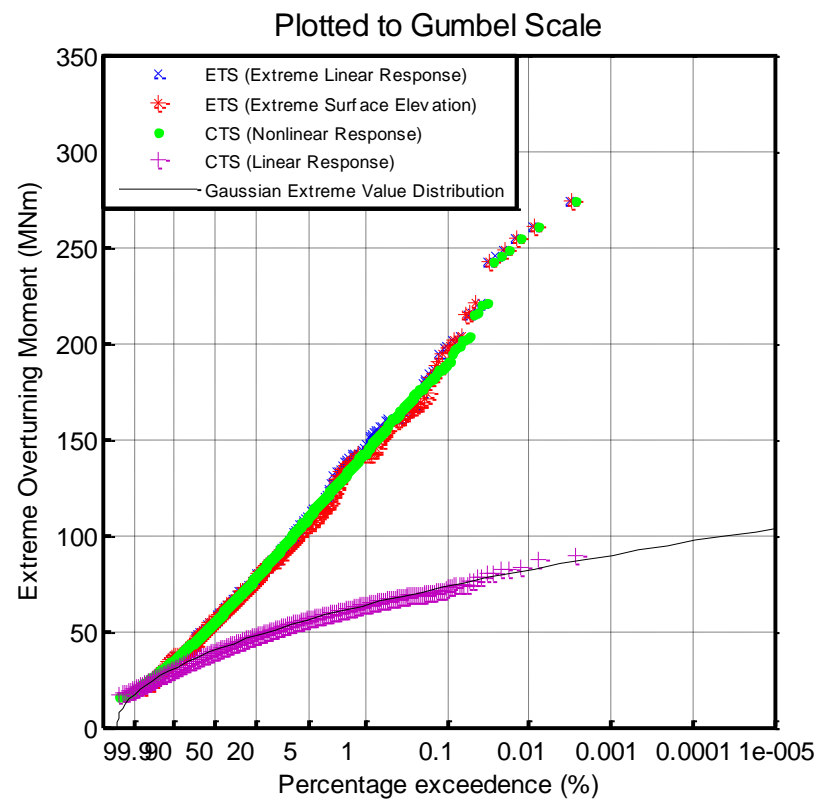

Fig. (9). Comparison of short-term probability distribution of extreme values of quasi-static overturning moment from ETS methods (Case 1, 220 simulations) with corresponding distribution from CTS (20000 simulations), $H_{s}=10 \mathrm{~m}, T_{z}=11.23 \mathrm{sec}, T=128 \mathrm{~s}$.

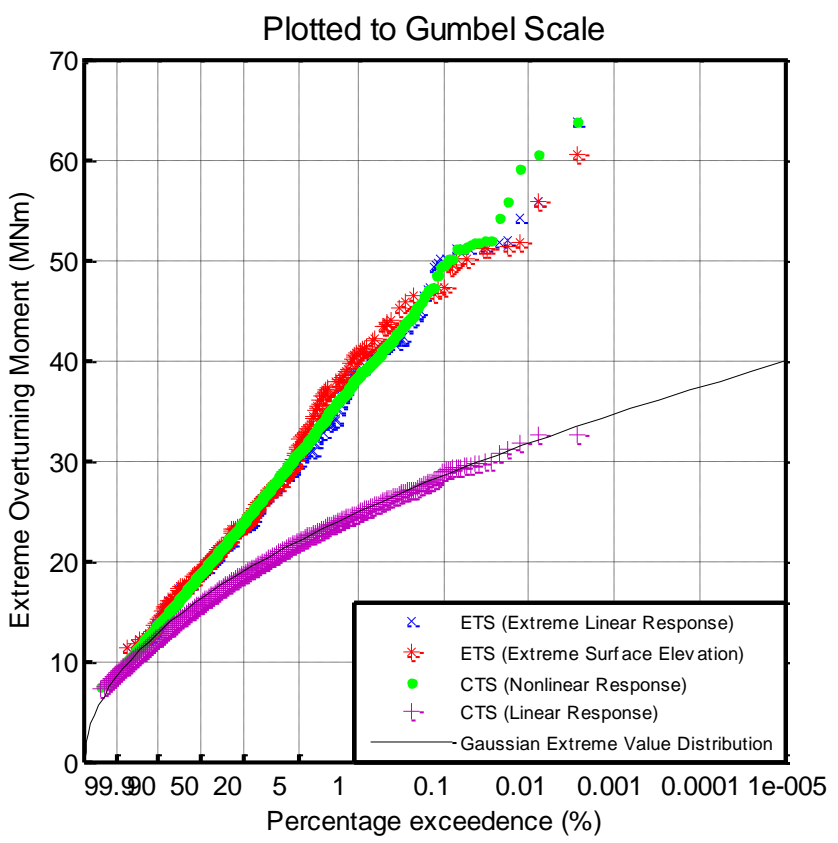

Fig. (10). Comparison of short-term probability distribution of extreme values of quasi-static overturning moment from ETS method (Case 1, 220 simulations) with corresponding distribution from CTS (20000 simulations), $H_{s}=5 \mathrm{~m}, T_{z}=7.94 \mathrm{sec}, T=128 \mathrm{~s}$.

response extreme values (using the assumption that the linear response is Gaussian). As observed, the effect of drag nonlinearity is very important especially at low probabilities of exceedence even for the case of $H_{s}=5 \mathrm{~m}$, indicating that the linear assumption is unsafe.

In terms of computational demand, the ETS method is about $(20000 / 220 \approx) 90$ times more efficient than the CTS method. It should, however, be recognised that the computa-

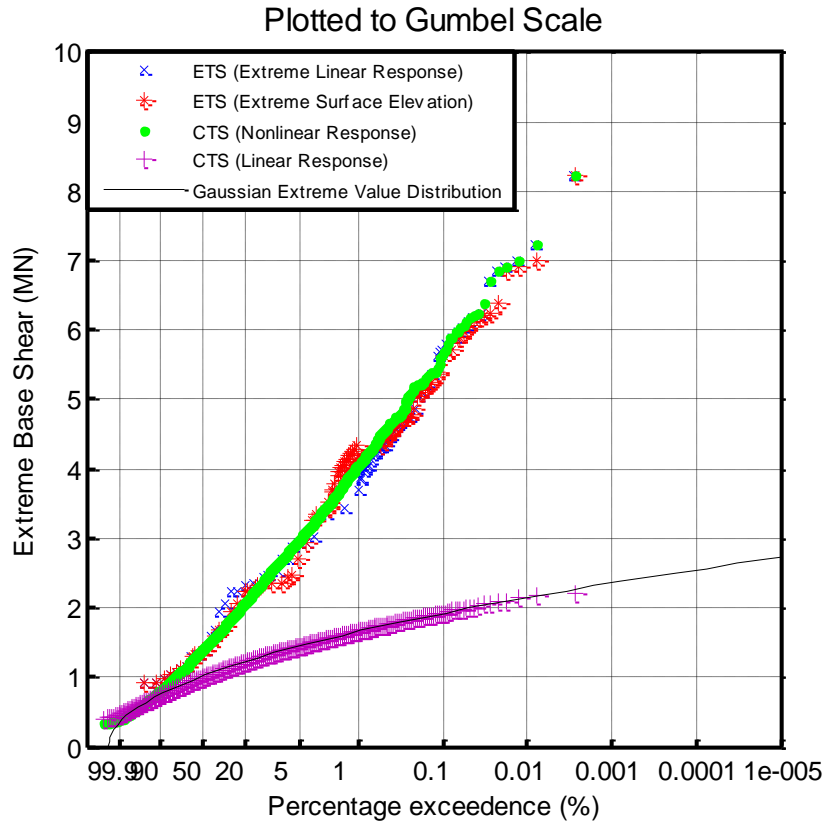

Fig. (11). Comparison of probability distribution of extreme values of quasi-static base shear from ETS method (Case 2, 100 simulations) with corresponding distribution from CTS (20000 simulations), $H_{s}=15 \mathrm{~m}, T_{z}=13.75 \mathrm{~s}, T=128 \mathrm{~s}$.

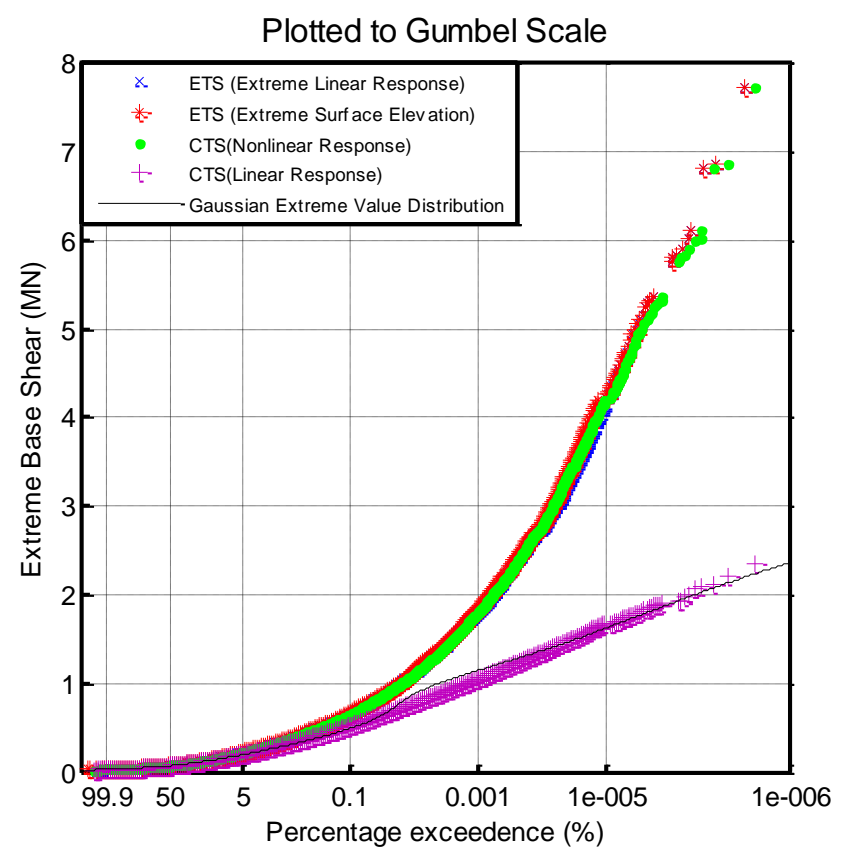

Fig. (12). Comparison of long-term probability distribution of extreme values of quasi-static base shear from ETS methods with corresponding distribution from CTS method.

tional effort for the ETS method can be improved by further reducing the number of simulated response records allocated to each group. For example, the probability distribution of the extreme values of quasi-static base shear for Case 2 (based on a total number of 100 simulated response extreme values, refer to Table 6, Column 5) is shown in Fig. (11). As observed, even with such a small number of simulations, a reasonably accurate estimate of the probability distribution of response extreme values can be obtained. 
Table 7. Comparison of Extreme Response Values at 0.1 Percentage of Exceedence from Efficient Time Simulation (ETS) and Conventional Time Simulation (CTS) Techniques

\begin{tabular}{|c|c|c|c|c|c|c|}
\hline \multirow{2}{*}{ Method } & \multicolumn{2}{|c|}{$\boldsymbol{H}_{s}=\mathbf{1 5 m}$} & \multicolumn{2}{c|}{$\boldsymbol{H}_{s}=\mathbf{1 0 m}$} & \multicolumn{2}{c|}{$H_{s}=\mathbf{5 m}$} \\
\cline { 2 - 7 } & BS (MN) & OTM (MNm) & BS (MN) & OTM (MNm) & BS (MN) & OTM (MNm) \\
\hline \hline ETS (Extreme Surface Elevation) & 5.598 & 500.60 & 2.063 & 197.69 & 0.502 & 47.50 \\
\hline ETS (Extreme Linear Response) & 5.627 & 504.83 & 2.019 & 195.93 & 0.519 & 50.27 \\
\hline CTS & 5.622 & 503.11 & 2.033 & 192.70 & 0.515 & 49.60 \\
\hline
\end{tabular}

Note: BS: Base shear, OTM: Overturning Moment, ETS: Efficient Time Simulation and CTS: Conventional Time Simulation

Table 8. Level of Accuracy of Extreme Response Values at 0.1 Percentage of Exceedence from Surface Elevation and Linear Response Versions of the ETS Technique

\begin{tabular}{|c|c|c|c|c|c|c|}
\hline Sea state & \multicolumn{2}{|c|}{$H_{s}=\mathbf{1 5 m}$} & \multicolumn{2}{c|}{$H_{s}=\mathbf{1 0 m}$} & \multicolumn{2}{c|}{$H_{s}=\mathbf{5 m}$} \\
\hline Ratio $=$ ETS/CTS & BS (MN) & OTM (MNm) & BS (MN) & OTM (MNm) & BS (MN) & OTM (MNm) \\
\hline \hline ETS (Extreme Surface Elevation) & 0.9957 & 0.9950 & 1.0148 & 1.0259 & 0.9748 & 0.9577 \\
\hline ETS (Extreme Linear Response) & 1.0009 & 1.0034 & 0.9931 & 1.0168 & 1.0078 & 1.0135 \\
\hline
\end{tabular}

Note: BS: Base shear, OTM: Overturning Moment, ETS: Efficient Time Simulation.

Table 9. Ratios between Corresponding Standard Deviations of Inertia-induced and Drag-induced Components of Overturning Moment. Sample Record Duration $=32768$ Seconds

\begin{tabular}{|c|c|c|c|}
\hline Sea State & $\boldsymbol{\sigma}_{\text {Inertia }}$ & $\boldsymbol{\sigma}_{\text {Drag }}$ & Ratio $=\frac{\boldsymbol{\sigma}_{\text {Inertia }}}{\boldsymbol{\sigma}_{\text {Drag }}}$ \\
\hline \hline$H_{s}=15 \mathrm{~m}$ & 251.4076 & 386.3491 & 0.6507 \\
\hline$H_{s}=10 \mathrm{~m}$ & 168.5053 & 150.8957 & 1.1167 \\
\hline$H_{s}=5 \mathrm{~m}$ & 72.9542 & 29.2168 & 2.4970 \\
\hline
\end{tabular}

Note: $\sigma_{\text {Inertia: }}$ Standard deviation of inertia-induced component, $\sigma_{\text {Drag: }}$ Standard deviation of drag-induced component.

\section{COMPARISON OF THE TWO ALTERNATIVE VERSIONS OF THE ETS PROCEDURE: LONG- TERM PROBABILITY DISTRIBUTION OF RE- SPONSE EXTREME VALUES}

The long-term probability distribution of the extreme values of nonlinear quasi-static base shear accounting for all the sea states in the scatter diagram (Table 1) is shown in Fig. (12). As observed, both versions of ETS method are in very good agreement with corresponding distribution from the CTS procedure. The linear response version is, however, more accurate. Again, the effect of nonlinearity is evident by comparing the distribution of the nonlinear response extreme values with corresponding distribution of the linear response extreme values. The effect is particularly prominent at low probabilities of exceedence.

\section{CONCLUSIONS}

- The conventional time simulation (CTS) procedure is frequently used for derivation of the probability distribution of the extreme values of offshore structural response due to wave and current loading. However, this procedure is computationally very demanding as a large num- ber of simulations is required to reduce the sampling variability to acceptable levels.

- An alternative to the CTS procedure was recently introduced (ETS), which divides the simulated response extreme values into a number of groups based on the magnitude of the extreme values of their associated surface elevation records. The probability distribution of response extreme values for each group is then calculated individually based on a relatively small number of simulations, and then the total probability theorem is used to derive the probability distribution of response extreme values.

- In this paper, a new version of the ETS technique has been introduced, which takes advantage of the good correlation between the linear and nonlinear response extreme values.

- The two versions of the ETS procedure have been tested by comparing the short-term probability distributions of the response extreme values from them with corresponding distributions from the CTS method (based on a very large number of simulated extreme values). Both versions of the ETS method are good but the linear response 
version is somewhat better, especially for low-intensity sea states.

- The two versions of the ETS procedure have also been tested by comparing the long-term probability distributions of the response extreme values from them with corresponding distributions from the CTS method. Both versions of the ETS method are very good but the linear response version is somewhat better.

- In this study, the validation of the ETS procedure has been limited to quasi-static responses in the absence of current. The general case of a dynamic structure exposed to combined wave and current loading is under investigation.

\section{CONFLICT OF INTEREST}

The author(s) confirm that this article content has no conflicts of interest.

\section{ACKNOWLEDGEMENTS}

This paper is financially supported by the Ministry of Higher Education (Malaysia) and Universiti Teknologi Malaysia (UTM) which is gratefully acknowledged.

\section{REFERENCES}

[1] A. Naess, O. Gaidai and S. Haver, "Efficient estimation of extreme response of drag-dominated offshore structures by Monte carlo simulation". Applied Ocean Research, vol 34, no. 16, pp. 2188219, 2007.

[2] G. Najafian, "Probability models for offshore structural response due to Morison wave loading, Part I: Drag-only response". Ocean Engineering, vol. 34, no. 17-18, pp. 2277-2288, 2007.

[3] G. Najafian, "Application of system identification techniques in efficient modeling of offshore structures, Part I: model development". Applied Ocean Research, vol. 29, no. 1-2, pp. 1-16, 2007.

[4] L. E. Borgman, "Random hydrodynamic forces on objects". The Annals of Mathematical Statistics, vol. 38, pp. 37-51, 1967.

[5] R. G. Tickell, "Continuous random wave loading on structural members". The Structural Engineer, vol. 55, no. 5, pp. 209-22, 1977.

[6] R. Burrows, "Probabilistic description of the response of offshore structures to random wave loading". In: Shaw TL, Ed. Mechanics of Wave Induced Forces on Cylinders. London: Pitman Advanced Publishing Program; pp. 577-95, 1979.
[7] T. Eatock, and A. Rajagopalan, "Super harmonic resonance effects in drag dominated structures". In: Proceedings of the $2^{\text {nd }}$ international symposium on integrity of offshore structures. pp. 85-104, 1981.

[8] C. C. Tung, , "Effect of free surface fluctuation on total wave force on cylinder", Journal of Engineering Mechanics, ASCE, vol. 121, no. 2, pp. 274-280, 1995.

[9] C. Y. Liaw, and X. Y. Zheng, , "Polynomial approximations of wave loading and super harmonic responses of fixed structures", Journal of Offshore Mechanics and Arctic Engineering, ASME, vol. 25 , no. 3, pp. 161-167, 2003.

[10] M. K. Abu Husain, and G. Najafian, "An efficient Monte Carlo simulation technique for derivation of the probability distribution of the extreme values of offshore structural response", In: Proceedings of the $29^{\text {th }}$ International Conference on Offshore Mechanics and Arctic Engineering, Shanghai, China, pp. 1-7, 2010.

[11] W. . Pierson and L. Moskowitz, "A Proposed Spectral Form for Fully Developed Wind Seas Based on the Similarity Theory of S.A. Kitaigorodskii", Journal of Geophysical Research, vol. 69, pp. 5181-5190, 1964.

[12] T. Sarpkaya, and M. Isaacson, "Mechanics of Wave Forces on Offshore Structures", Van Nostrand Reinhold, New York 1981.

[13] G. Moe, and R. L. P. Verley, "Hydrodynamic damping of offshore structures in waves and currents", Proceedings of the OTC, pp. 3744, 1980.

[14] L. E. Borgman, "Ocean wave simulation for engineering design". ASCE Journal of Waterways and Harbours Division, vol. 95(WW4), pp. 557-83, 1969.

[15] C. K. Marooka, and I. H. Yokoo, "Numerical simulation and spectral analysis of irregular sea waves", Journal of Offshore and Polar Engineering, pp.189- 196, 1997.

[16] M. J. Tucker, D. J. T. Carter, and P. G. Challenor, "Numerical simulation of random sea: A common error and its effect upon wave group statistics", Applied Ocean Research, vol. 29, no. 6, pp. 118-122, 1984.

[17] A. T. Couch and J. P. Conte, , "Field verification of linear and nonlinear hybrid wave models for offshore tower response prediction". Journal of Offshore Mechanics and Arctic Engineering, vol. 119, pp. 158-65, 1997.

[18] P. W. Marshall, and R. B. Inglis, , "Wave Kinematics and Force Coefficients". ASCE Structures Congress, 1986.

[19] R. B. Inglis, J. G. L. Pijfers. and J. H. Vugts, "A unified probabilistic approach to predicting the response of offshore structures, in cluding the extreme response". Proceeding of the 4th International Conference on the Behaviour of Offshore Structures, Delft, Netherland. pp. 95-109, 1985.

[20] M. J. Tucker, "Waves in Ocean Engineering: Measurement, Analysis, Interpretation”. Ellis Herwood, London, 1991.

[21] R. Torhaug, S. R. Winterstein, and A.Braathen, , "Nonlinear ship loads: stochastic models for extreme response". Journal of Ship Research, vol. 42, no. 1, pp.46-55, 1998. 